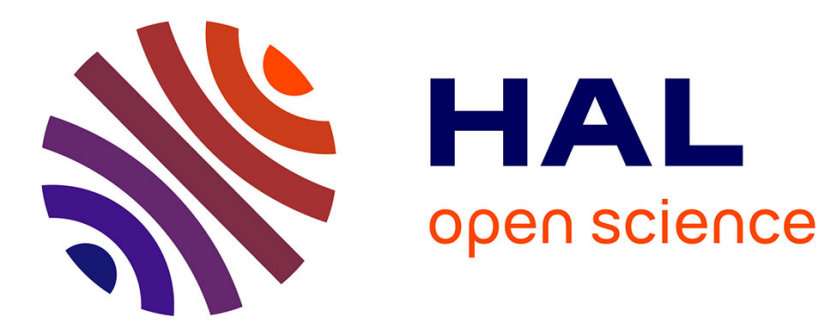

\title{
Ziyad Rahbani, une figure inédite de l'homme de théâtre au Liban
}

Arnaud Chabrol

\section{To cite this version:}

Arnaud Chabrol. Ziyad Rahbani, une figure inédite de l'homme de théâtre au Liban. Franck Mermier et Nicolas Puig. Itinéraires esthétiques et scènes culturelles au Proche-Orient, Presse de l'Institut français du proche-Orient, pp.77-99, 2007. halshs-00639017

\section{HAL Id: halshs-00639017 https://shs.hal.science/halshs-00639017}

Submitted on 7 Nov 2011

HAL is a multi-disciplinary open access archive for the deposit and dissemination of scientific research documents, whether they are published or not. The documents may come from teaching and research institutions in France or abroad, or from public or private research centers.
L'archive ouverte pluridisciplinaire HAL, est destinée au dépôt et à la diffusion de documents scientifiques de niveau recherche, publiés ou non, émanant des établissements d'enseignement et de recherche français ou étrangers, des laboratoires publics ou privés. 


\section{Ziyad Rahbani, une figure inédite de l'homme de théâtre au Liban}

Arnaud Chabrol

\section{Introduction}

D'aucuns pourraient s'étonner, en lisant cet article, que Ziyad Rahbani y soit considéré d'emblée en tant qu'homme de théâtre. En effet, au vu de son parcours, cet artiste libanais semble davantage tourné vers la musique. Une étude réalisée récemment sur son public montre pourtant que ce dernier découvre l'artiste par le biais du théâtre ${ }^{1}$. Bien que cela puisse paraître paradoxal, quand on connaît l'omniprésence de sa musique sur les ondes libanaises (musique et chansons, nouvelles ou anciennes, y sont quotidiennement diffusées) et son style singulier au sein de la production musicale libanaise et arabe, cela justifie partiellement notre perspective. De plus, malgré son profil marginal dans le paysage du théâtre libanais contemporain, Ziyad Rahbani en est une figure incontournable. Les œuvres politiques qu'il a mises en scène au cours de la guerre civile (1975-1990) ont acquis un statut particulier au sein de la production dramatique nationale, mais aussi dans la société libanaise. Resituer son œuvre dans son contexte de production permet donc à la fois de mieux comprendre la place de cet artiste et de son œuvre dans le Liban contemporain et de saisir, à partir d'une figure excentrée du champ théâtral, le fonctionnement de ce dernier.

$\mathrm{Au}$ Liban comme dans le reste du monde arabe, le théâtre est une discipline artistique récente. La seule tradition théâtrale connue est le théâtre rituel chiite qui met en scène la mort de l'imam Husayn dans l'épopée de Karbala ${ }^{2}$. Le théâtre, dans sa forme occidentale, est introduit à Beyrouth en 1848 à l'initiative d'un riche marchand beyrouthin féru de littérature, Marûn

1. Kamal Abou Ghaida 2002.

2. Connu en Occident sous le nom de ta 'ziyeh, emprunté au Persan. Voir l'article de Sabrina Mervin dans ce volume. 
al-Naqqâsh. Sa mise en scène d'un Avare « entièrement chanté sur le mode des Maqams traditionnels ${ }^{3}$ » va influencer l'ensemble de la production arabe durant plus d'un siècle ${ }^{4}$. Les adaptations les plus populaires du répertoire occidental ou de récits historiques locaux sont ainsi accompagnées par de la musique orientale interprétée par un orchestre et un chœur. Au Liban, le théâtre des frères Rahbani ('Assi et Mansour) est exemplaire de cette tendance. Outre l'enthousiasme qu'elle suscite, leur œuvre pléthorique revêt une importance particulière, participant pleinement à la construction d'une identité libanaise au lendemain de l'indépendance. Danses, costumes et personnages folkloriques symbolisent la vie du Liban traditionnel ; la musique, la poésie et les chants en dialecte libanais prennent largement le dessus sur des textes dont la trame paraît souvent décousue. Pour les frères Rahbani, le théâtre semble surtout avoir été un prétexte où l'espace rendait possible la mise en scène de leurs découvertes musicales et poétiques. C'est peut-être pour cela que leur théâtre reste en marge du « mouvement théâtral libanais ${ }^{5}$ ».

Ce dernier naît dans le prolongement de la révolution poétique arabe de la fin des années 1950. Regroupée à Beyrouth autour de la revue Shi 'r (Poésie), l'avant-garde littéraire soutient le mouvement à ses débuts. Cependant, les hommes de théâtre libanais affirment rapidement ce qui a été décrit en Europe comme le « primat de la scène ${ }^{6}$ » et détournent progressivement la composition des textes de son caractère littéraire. Le mouvement théâtral est en outre profondément influencé par le choc de la défaite arabe de 1967 qui amorce un virage radical dans les conceptions de l'art en général.

Ziyad Rahbani apparaît sur la scène libanaise au début des années 1970 . Il semble alors être à la fois dépositaire d'un héritage familial imposant (celui du Liban traditionnel glorifié par le théâtre des frères Rahbani) et témoin des crises sociales et politiques que traverse le Liban moderne. Sa carrière est particulièrement éclairante sur une période qu'on peut qualifier de « transitoire » pour le mouvement théâtral.

3. KHOURY 1999, p. 91.

4. C'est cependant en Égypte que le théâtre connaîtra son essor jusqu'au début du $\mathrm{Xx}^{\mathrm{e}}$ siècle. Cet essor s'explique essentiellement par deux facteurs : d'une part, la place privilégiée qu'accorde le Khédive Ismaïl à la culture occidentale, concrétisée notamment par sa commande à Verdi d'une œuvre (Aïda) interprétée en 1869 à l'occasion de l'inauguration de l'Opéra du Caire ; d'autre part, les comédiens et artistes fuyant les restrictions (voire persécutions dans le cas de Abû-Khalîl Qabbânî) auxquelles il étaient sujets à Beyrouth et Damas trouvent refuge au Caire et apportent avec eux leurs conceptions du théâtre.

5. Cette dénomination, traduite de l'arabe (al-Harakat al-masrahiyya), désigne aujourd'hui une production dramatique dans son ensemble (sans considération de genre ni d'époque). L'expression n'en reste pas moins fortement liée au contexte libanais des années 1960, à la naissance d'un « théâtre d'art »au Liban. C'est à cette première occurrence que nous nous référons dans l'article.

6. Proust 2001, p. 472. 
Des années 1960 à la guerre civile, le théâtre contemporain libanais connaît une première phase de structuration. La période est également caractérisée par la politisation et la radicalisation des milieux artistiques. En conséquence, la carrière des hommes de théâtre libanais est fortement influencée par un répertoire de pratiques sociales lié au militantisme politique. « Le travail social de définition et de délimitation ${ }^{7}$ » de l'activité théâtrale auquel se consacre cette nouvelle catégorie d'artistes est en outre entravé par les tensions qui agitent le Liban au tournant des années 1970. Ainsi, à travers l'étude de la carrière de Ziyad Rahbani, son positionnement et sa plus ou moins grande intégration vis-à-vis de la scène théâtrale libanaise, transparaît en filigrane un « état » du champ théâtral libanais contemporain.

Après avoir présenté de manière générale l'évolution des arts scéniques dans le contexte des années 1970, nous verrons en effet que la trajectoire professionnelle de Ziyad Rahbani aide à comprendre comment l'engagement politique favorise la reconnaissance de nouveaux artistes, le plus souvent non professionnels, par les milieux artistiques. Cependant, si Ziyad Rahbani a effectivement, mais pas exclusivement, intégré le champ du théâtre libanais sur la base d'une communauté de principes éthiques avec les milieux artistiques, l'esthétique simpliste de son théâtre et sa position ambiguë au sein de la production culturelle nationale l'ont indéniablement marginalisé en tant qu'homme de théâtre.

Le cadre de cet article ne permet pas de rendre compte de l'essor qu'a connu la pratique du théâtre au cours des années 1960. Par conséquent, notre objet d'étude se restreint ici à quelques points. Ainsi, nous nous appuyons exclusivement sur la production arabophone, essentiellement parce qu'elle est la plus prolifique. De plus, nos analyses se fondent sur l'observation des pratiques artistiques qui nous semblent illustrer avec justesse les positions esthétiques et idéologiques dominantes dans les arts scéniques au cours de cette période. Enfin, nous avons délibérément exclu les deux dernières œuvres de Ziyad Rahbani (représentées respectivement en 1993 et $1994{ }^{8}$ ), car elles n'appartiennent pas à la période traitée dans cet article.

C'est par ailleurs à dessein que cette étude rompt avec les lectures internes de l'œuvre de Ziyad Rahbani, ces dernières privilégiant trop souvent son caractère politique au détriment des conditions sociales de sa production. Aussi, afin de restituer l'œuvre « dans des conditions pratiques d'existence sociales et économiques [...] et dans les conditions matérielles et temporelles ${ }^{9} \gg$ de sa

7. BOLTANSKI 1982, p. 52.

8. Il s'agit de Bi khușūṣ al-karāma wa al-sha 'b al- 'anìd (De la dignité et du peuple têtu, 1993, non publiée) et Law lā fushat al-amal (Si ce n'est une lueur d'espoir, 1994, non publiée).

9. LAHIRE 2006, p. 11. 
création, inscrivons-nous notre analyse dans la mouvance de la sociologie de la production culturelle. Cette perspective permet d'une part d'appréhender les conditions et les enjeux de la naissance d'un art nouveau (le théâtre) au sein de l'espace culturel libanais ; et, d'autre part, favorise la compréhension des processus socio-politiques qui ont affecté cet espace, notamment après la défaite arabe de 1967.

\section{Politisation du champ de production culturel}

\section{Contexte social et historique}

Ziyad Rahbani apparaît sur la scène artistique à l'apogée d'un long processus de politisation de la société libanaise. Au cours des années 1960, le Liban voit émerger de nouvelles formes d'expression politique qui se cristallisent notamment dans la naissance d'un syndicalisme étudiant ${ }^{10}$. Par ailleurs, le traumatisme causé par la Naksa ${ }^{11}$ vient ranimer un ensemble de mises en question. Cette période se caractérise par l'effervescence d'une intelligentsia radicalisée et une forte mobilisation des milieux intellectuels et étudiants qui, selon Nadine Picaudou, s'opère autour de trois pôles : « soutien inconditionnel à la cause palestinienne, revendications de justice sociale, exigences de démocratisation et déconfessionnalisation du système politique ${ }^{12}$. "

De plus, les créations et dissolutions successives d'un grand nombre de cercles et d'associations partisanes engendrent, dès le début des années 1970, des repositionnements plus ou moins radicaux de la part des militants. Deux types d'engagement nous semblent particulièrement intéressants dans le cadre de cette étude. Le plus radical, celui des opérations armées contre des cibles étrangères ou libanaises, reste marginal dans le faisceau des pratiques militantes, mais a été une source d'inspiration pour la première œuvre politique de Ziyad Rahbani ${ }^{13}$. Le second concerne une majorité de militants et consiste en un engagement direct et individuel dans une organisation palestinienne. Plusieurs intellectuels et artistes expriment ainsi leur soutien à la lutte armée ${ }^{14}$.

10. FAVIER 2004.

11. Défaite des armées arabes face à Israël en 1967.

12. Picaudou 1989, p. 128.

13. La pièce Nazil as-surūr (La Pension du bonheur, 1974, 1993) s'inspire de la prise d'otages de la Bank of America en 1974 : DAKROUB et SAAD 1982. Le commando justifie alors son action par la « nécessité de l'expropriation prolétarienne ».

14. Ce sera notamment le cas de Roger Assaf (acteur et metteur en scène) et de Élias Khoury (écrivain). Sur le lien entre la radicalisation des pratiques militantes et l'instabilité des organisations partisanes, voir FAVIER 2004, p. 320. 
L'effervescence politique est aussi perceptible dans le contenu de la production culturelle au sens large. Ce d'autant que Beyrouth attire les exilés arabes de tous bords dont l'affluence accompagne par vagues les crises et durcissements auxquels sont en proie les régimes arabes voisins ${ }^{15}$. Cette présence arabe plurielle ${ }^{16}$ fortement politisée stimule, entre autres, le secteur éditorial libanais. Ce dernier, assumant pleinement son rôle d'intermédiaire entre auteurs et public, devient « un vecteur important des débats idéologiques constituant Beyrouth comme un creuset de la production intellectuelle arabe ${ }^{17}$. " Voyant dans ce secteur un moyen efficace d'élargir leurs réseaux d'influence, les organisations partisanes d'obédiences variées font de l'édition l'outil privilégié de leur propagande.

La multiplication des revues culturelles et leur contenu témoignent aussi de l'emprise de la politique sur le champ culturel. La surdétermination des options esthétiques par les clivages idéologiques se renforce au fur et à mesure qu'augmentent les tensions sur le territoire. Elle affecte d'autant plus les arts « nouveaux » que ceux-ci attirent, dans leur quête d'autonomie ${ }^{18}$, de nouveaux entrants dans le champ de production culturel.

\section{Options idéologiques et arts scéniques}

La scène libanaise accueille des pratiques nouvelles, d'ores et déjà acquises à la «cause révolutionnaire ». C'est l'âge d'or du théâtre et de la chanson politiques. Même si, selon l'artiste libanais Abidou Basha, «le désir d'expression est alors plus important que sa forme ${ }^{19} »$, la recherche

\footnotetext{
15. PICARD 1988.

16. La diversité des populations arabes de Beyrouth transparaît tant dans le nombre de nationalités qui s'y côtoient que dans l'hétérogénéité des profils qu'elles drainent dans leur sillage.

17. Mermier 2005, p. 57.

18. Pierre Bourdieu définit le champ comme un microcosme régi par des règles et des enjeux propres, irréductibles à ceux des autres champs. Un champ reste cependant soumis à des contraintes qui lui sont étrangères, exercées par des champs englobants ou concurrents tels que ceux du pouvoir politique ou économique. C'est à ces derniers que renvoie la notion d'autonomie. L'enjeu spécifique qui caractérise l'autonomie du champ culturel réside dans la rémunération symbolique que constitue la reconnaissance des pairs, ce qui permet au sociologue de dire que le champ culturel se présente comme un « monde économique renversé » dans lequel les gratifications matérielles peuvent être perçues comme un rapprochement avec des valeurs étrangères au champ (art commercial) et où, par conséquent, « ceux qui y entrent ont intérêt au désintéressement », Bourdieu 1992, p. 353.

19. Basha 2005, p. 34. Abidou Basha est un artiste libanais dont l'engagement débute au tournant des décennies 1970 et 1980. L'expérience qu'il relate dans son ouvrage Mort d'un directeur de théâtre constitue un témoignage important sur les modalités de l'engagement politique des artistes libanais. Il y insiste notamment sur les croyances partagées, les valeurs et les enjeux de la création dans les domaines de la musique et du théâtre ainsi que sur les multiples réseaux militants qui se tissent et organisent les pratiques au cours de cette période.
} 


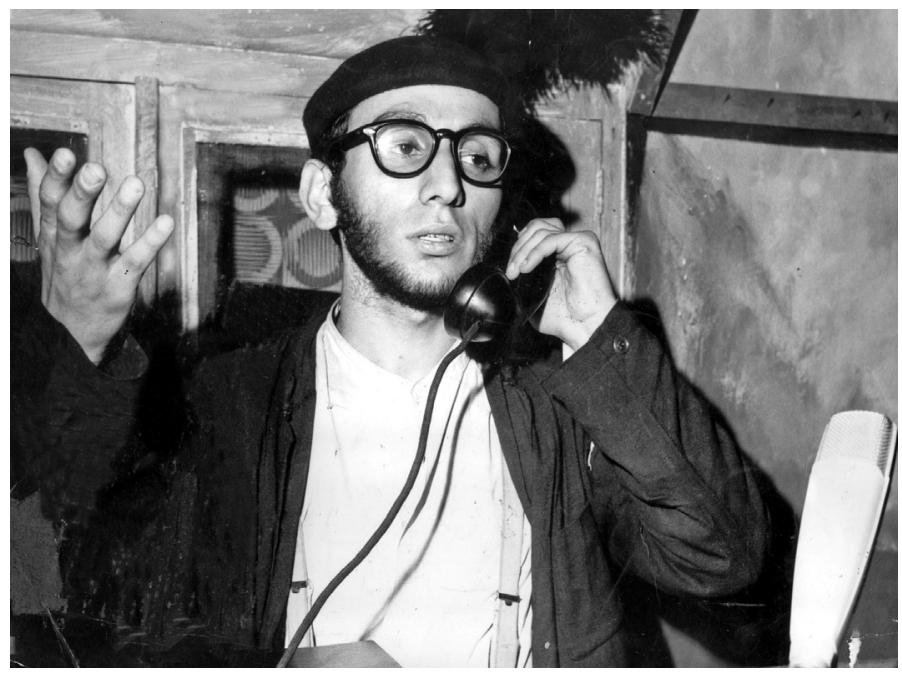

Ziyad Rahbani dans Nazil al-surūr (1974). (C) Dār al-mukhtarāt.

esthétique reste un enjeu au sein du champ de production artistique. En effet, selon une logique propre aux champs artistiques, on constate que malgré la prégnance de la politique dans le domaine de l'art, les prises de position des artistes continuent d'obéir « à des logiques qui n'ont pas la politique pour seul principe ${ }^{20} »$. Au Liban, « l'émergence de la chanson politique ${ }^{21}$ » reflète bien cet engouement pour la politisation des arts ; ce genre musical est d'ailleurs organiquement lié au mouvement théâtral.

Ainsi, Abidou Basha situe la naissance de la chanson politique en 1971, année qui voit la création de deux pièces au cours desquelles seront diffusées des chansons à contenu politique. Il s'agit de l'adaptation de La résistible ascension d'Arturo Ui de Bertolt Brecht par le metteur en scène Jalal Khoury et de la pièce Majdalūn (1969), créée par l'Atelier dramatique de Beyrouth (muhtaraf Bayrūt li-l-masrah) ${ }^{22}$. Abidou Basha, Paul Matar, Sami Hawat et Marcel Khalifeh, interprètes ou compositeurs de ce genre musical nouveau, accompagnent (à des degrés différents) l'évolution des deux formes d'art.

20. SAPIRO 1999, p. 12.

21. " The rise of the political song », BARDAWIL 2002, p. 18.

22. Basha ajoute que « le lien qui unit la chanson au théâtre remonte aux obsessions de cette étape : la rébellion contre l'appareil à la recherche d'un système [politique] nouveau. » BASHA 2005, p. 30. La pièce de Khoury est montée sous le titre de Wayzmānū, Bin Ghūryūn wa shurakā'hu (Weizmann, Ben Gourion et Cie, 1968). L'Atelier dramatique de Beyrouth est fondé en 1968 par les comédiens Nidal al-Ashqar et Roger Assaf. Les ambitions affichées sont alors clairement politiques. La représentation de la pièce Majdalūn sera interrompue manu militari par les autorités et les comédiens arrêtés alors qu'ils tentaient de prolonger la représentation dans un café de la rue Hamra (le Horseshoe). 
Ziyad Rahbani, quant à lui, compose de nombreuses chansons pour ses propres pièces avant de s'affirmer plus clairement dans cette mouvance avec l'album Anā mush käfir (Je ne suis pas un mécréant, 1985).

Enfin, chansonniers et hommes de théâtre partagent un certain nombre de principes. D'une part, leur pratique de l'art participe clairement d'un projet politique. D'autre part, leur volonté de rupture avec le mode de production dominant se cristallise dans un effort de création collective. Cette dernière s'avère fondamentale dans le processus de politisation des deux pratiques artistiques. Elle répond au besoin prioritaire de contrecarrer la culture de masse assimilée aux « formes sur lesquelles s'appuie la bourgeoisie arabe pour distraire le peuple de ses problèmes essentiels » et qui encouragent l'union des classes au détriment de leur lutte ${ }^{23}$. Cependant, si dans le cas de la musique, la création collective vise en partie à supplanter le système du vedettariat, dans le cas du théâtre, elle participe d'un processus antérieur et plus complexe de mise en question des principes du théâtre dominant, considéré comme 《bourgeois » ${ }^{24}$. Le processus de politisation du mouvement théâtral libanais reste donc dans une large mesure passible d'une analyse propre visant à souligner la spécificité de ses enjeux.

\section{Théâtre et politique}

En 1974, dans le mémoire de maîtrise qu'il a consacré au Théâtre politique au Liban, Ghassan Salamé soulignait que « la naissance du théâtre libanais est un fait colonial ${ }^{25}$ ». Le développement du mouvement théâtral libanais prend ainsi rapidement la forme d'une quête identitaire. Celle-ci vouait en quelque sorte le théâtre à se tourner vers la politique. De plus, la convergence de facteurs hétérogènes tels que la recherche d'authenticité et la crise sociale, auxquels s'ajoute le désarroi de la défaite de 1967, affecte profondément sa trajectoire. $\mathrm{Au}$ dire du metteur en scène Roger Assaf, cette défaite va paradoxalement « insuffler une nouvelle vie au théâtre libanais ${ }^{26}$ ».

À plusieurs égards, la politisation du théâtre libanais est des plus évidentes. En premier lieu, la mise en scène de l'histoire ou de l'actualité permet d'aborder les questions politiques régionales et locales. Dans les deux cas, la fable s'appuie sur des événements et des personnages historiques précis.

23. Dans le domaine de la musique, cette critique se fait particulièrement sentir à l'encontre du concept de tarab (extase musical) autour duquel se cristallisent les tensions consécutives aux tentatives de définition des objectifs de la chanson politique. BASHA 2005, p. 73-80.

24. Assaf 1984 ; Salamé 1974.

25. SAlAmÉ 1974, p. 12.

26. AssAF 2000. 
Cette quête de réalisme est d'autant plus marquée que la quasi-totalité de la production s'inspire des théories brechtiennes visant à établir le théâtre comme moyen d'exploration de la réalité sociale.

En second lieu, les membres du mouvement théâtral sont pour la plupart affiliés à une organisation politique. Qu'ils soient membres d'une organisation partisane ou simples sympathisants, leur lecture de l'histoire s'en trouve clairement affectée. Au sein de l'Atelier dramatique de Beyrouth, plusieurs acteurs sont ainsi membres du Parti social nationaliste syrien ${ }^{27}$. Ghassan Salamé note que, dans la mesure où les textes mis en scène par l'Atelier sont élaborés à partir d'un travail d'improvisation collective, ceux-ci sont très certainement sous-tendus par l'idéologie de ce parti. Jalal Khoury, metteur en scène et membre du Parti communiste libanais (PCL), considéré comme « le brechtien orthodoxe ", voit quant à lui dans l'application stricte des préceptes du dramaturge allemand un moyen efficace de diffuser une lecture marxiste du conflit israélo-palestinien. Ainsi, dans l'ensemble, la production dramatique reflète des obédiences idéologiques aisément identifiables, cristallisant parfois les querelles auxquelles leurs adeptes prennent part ${ }^{28}$.

Enfin, en marge de la production, les interprétations auxquelles se prêtent les œuvres et les mises en scène se font elles aussi l'écho des clivages idéologiques qui structurent le champ intellectuel libanais à cette époque. Comme le montre Ghassan Salamé, la critique soumet les œuvres dramatiques de l'époque à l'examen de leur forme scénique comme à celui de leur contenu politique. L'auteur n'échappe pas lui-même à cette tendance lorsqu'il dénonce chez deux hommes de théâtre « les aspects les plus incontestables de l'idéologie bourgeoise, consistant à nier le progrès historique au profit d'une vague loi de répétition et de récurrence, une vision pessimiste du déroulement de l'histoire $[\ldots]^{29}$. »

Ce contexte de forte politisation a un double impact sur la carrière de Ziyad Rahbani. D'une part, c'est son engagement qui le démarque radicalement de l'héritage des frères Rahbani. D'autre part, en s'affirmant en faveur d'un art politique, il assied sa carrière en tant qu'artiste indépendant dans différents domaines de la production artistique nationale.

27. Il s'agit de Rida Kibrit et de Nidal al-Ashqar dont le père est l'un des cadres dirigeants du parti.

28. SAlAmé 1974 , p. 89.

29. Salamé 1974, p. 55. Il s'agit du metteur en scène Yaacoub Chedraoui pour la création de al-Amir al-ạ̣mar (L'Émir rouge, 1973) et d'Antoine Ghandour pour sa pièce Akhwat Chanāy (Le fou de Chanay), montée en 1973 par Berj Vazélian. 


\section{Ziyad Rahbani et la production artistique libanaise}

\section{Un héritage investi}

Fils de Feyrouz et de 'Assi Rahbani, Ziyad entre très tôt dans le champ artistique libanais, débutant simultanément dans les domaines du théâtre et de la musique. Cette disposition symbolise avant tout l'investissement de son héritage artistique. Son père et son oncle sont tenus pour les précurseurs du genre de l'opérette au Liban et sa première participation à un travail de création trouve naturellement sa place dans la production familiale. Dans la pièce al-Mahatța (La Station, 1973), il joue du piano et est à la fois auteur et compositeur d'une chanson interprétée par Feyrouz, Sa 'alūnì al-nās (Les gens m'ont demandé, 1973). Cependant, son indépendance s'affirme cette même année. À l'âge de 17 ans, il met en scène sa première création, Sahriyya (Petite soirée, 1973). Malgré sa faible visibilité au sein de la production libanaise, cette pièce constitue son passeport pour le monde de l'art au Liban. D'une part, elle se situe dans la continuité de l'œuvre de sa famille (à l'instar des Rahabneh ${ }^{30}$, l'action se déroule dans un cadre rural et ne semble finalement qu'un prétexte pour une succession de compositions musicales). La pièce lui confère ainsi une parfaite légitimité dans le domaine de l'opérette. D'autre part, la figure de Ziyad y apparaît sous les traits qui seront désormais les siens : à la fois acteur, compositeur et auteur de ses pièces ${ }^{31}$.

Ce n'est qu'un an plus tard que Ziyad Rahbani fait son entrée sur la scène beyrouthine, lorsque, pour le cinquantième anniversaire de sa fondation, le PCL l'invite à remonter Sahriyya. À cette occasion, il mettra aussi en musique un hymne révolutionnaire écrit par Michel Soleiman, Yā riyāh $a l-s h a ' b$ (Tempête du peuple, 1973). Le rôle du parti communiste n'est pas anodin. En offrant une tribune à de nouveaux artistes, il fonctionne comme une instance de consécration parallèle à de nouvelles institutions comme la faculté des Beaux-Arts de l'Université libanaise, fondée en $1965^{32}$. Ce rôle légitimateur est plus évident encore dans le domaine de la musique engagée où les entrées ne sont pas alors sanctionnées par des critères de professionnalisme ${ }^{33}$. La

30. Nom donné à l'ensemble de l'œuvre des frères Rahbani.

31. Au Liban, Ziyad Rahbani est désigné par son prénom. C'est ce qui le distingue en premier lieu du reste de la famille et indique par ailleurs la perception que le public a de l'artiste : celle d'un artiste populaire. Cette image a de plus été largement diffusée par les médias et constitue encore une des caractéristiques distinctives de l'artiste.

32. La création de la faculté des Beaux-Arts, en 1965, permet entre autres de réguler les entrées dans le champ et devient rapidement un vivier d'acteurs dans lequel puisent les metteurs en scène.

33. Basha signale à plusieurs reprises le rôle de plus en plus imposant que jouent les organisations partisanes dans l'encadrement des activités culturelles. Au sujet du PCL, il parle notamment de « la famille des artistes du parti communiste » ('á'ilat fannānì al-hizb al-shuyū 'ì), BASHA 2005, p. 101. 
double inscription de Ziyad Rahbani dans les domaines de la musique et du théâtre lui permet de jouir d'emblée de la reconnaissance symbolique que lui confère son héritage. C'est cependant sa consécration au sein de la production artistique engagée qui lui octroie une légitimité propre qu'il ne cessera dès lors de revendiquer.

\section{Rupture et engagement}

Le deuxième temps qui ponctue l'entrée de Ziyad Rahbani dans le champ théâtral est la création de Nazil al-surūr (La pension du bonheur, 1974, 1993), moins d'un mois après le cinquantième anniversaire du PCL. C'est avec cette pièce qu'il consomme sa rupture avec l'œuvre théâtrale de ses parents. L'espace dramatique n'est plus rural mais urbain et bien que l'auteur ne précise pas le lieu de l'action, le spectateur reconnaît Beyrouth dès la scène d'exposition: une communication téléphonique fait part de la tenue d'une grève, les manifestations d'étudiants sont évoquées par recours aux bruitages et aux cris qui parviennent de la rue. La musique reste une composante importante du langage de l'auteur, mais elle ne perturbe pas la cohérence de la fable comme c'est le cas dans les Rahabneh et dans Sahriyya. Cependant, c'est surtout la satire de l'œuvre de ses parents dont on trouve les traces dès Nazil al-surūr qui marque la rupture. Elle révèle une tension avec la vision idyllique que les Rahbani présentent traditionnellement du patrimoine libanais. Cette critique allusive n'est dévoilée qu'à la fin de l'œuvre, mais n'en reste pas moins caractéristique de l'effort de distinction dont Ziyad Rahbani fait preuve en ce début de carrière ${ }^{34}$. Par ailleurs, cette œuvre concrétise scéniquement les orientations politiques de Ziyad Rahbani. La trame entretient un rapport direct avec l'actualité et présente les aspects contradictoires des modalités de l'engagement révolutionnaire et des attentes de la population. Avec Nazil alsurür, Ziyad s'ancre définitivement dans l'univers de la production artistique engagée.

Son engagement ne se matérialise pas uniquement dans sa pratique du théâtre. Un an après le déclenchement de la guerre civile, il s'établit à l'ouest de la capitale, en réaction à la chute du camp palestinien de Tall alZa'tar en $1976^{35}$. Ce nouvel ancrage géographique, dans une région où les

34. La pièce Shi fäshil (Un échec, 1982, 1993), constitue le point nodal de sa critique du contenu idéologique des Rahabneh.

35. Le siège du camp de Tall al-Za'tar a duré cinquante-deux jours. Sa reddition face aux milices chrétiennes marque la fin de l'époque où, aux yeux de la gauche libanaise, s'était opérée une forme de symbiose libano-palestinienne. « En un sens, dit Samir Kassir, c'était donc l'échec de tout le bouillonnement révolutionnaire des années soixante-dix. » La chute du camp entérine par ailleurs l'hégémonie des milices chrétiennes à l'est de la ville. KASsIR 1994, p. 226. 
organisations libanaises de gauche prédominent politiquement, est marqué par sa participation à une soirée musicale du West-hall, à l'Université américaine de Beyrouth. Cet événement réunit la plupart des artistes engagés auprès des organisations de gauche. La participation de Ziyad Rahbani, parce qu'elle représente « le mariage du positionnement politique avec la posture artistique et culturelle ${ }^{36} »$, suscite l'enthousiasme des participants. En outre, « le passage du fils de "Assi Rahbani et de Feyrouz du côté "pro-palestinien" confère une sorte de légitimité au Mouvement national ${ }^{37}$ », contredisant notamment la thèse, soutenue par la droite chrétienne, d'un conflit réduit à une bataille entre Libanais et Palestiniens.

Au cours de l'été 1976, Ziyad Rahbani débute la diffusion d'une émission de radio, Ba 'dnā tayyibin ... qul Allah! (Dieu soit loué, nous sommes toujours en vie!, 1976), en collaboration avec le cinéaste Jean Chamoun. L'audience déborde largement l'ouest de la capitale et le programme est écouté quotidiennement par une grande partie de la population beyrouthine. Au cours de l'émission, Ziyad Rahbani et Jean Chamoun commentent l'actualité à travers des sketchs corrosifs et fustigent tous les acteurs de la vie politique. De nombreux intellectuels et artistes sont aussi la cible de leurs sarcasmes. Ils sont généralement accusés de ne pas adhérer aux principes révolutionnaires ou d'être en contradiction avec ceux-ci, soit qu'ils mènent un mode de vie « bourgeois », soit que leur pratique de l'art ne s'adresse qu'à une élite, soit encore qu'ils aient quitté le pays au cours de la guerre civile ${ }^{38}$. Par contraste, cette dernière accusation octroie une forme de supériorité morale à Ziyad. Fadi Abdo Bardawil précise qu'à travers ses attaques répétées contre les acteurs des différents sous-champs du théâtre transparaissent en filigrane les caractéristiques de l'artiste engagé qui délimitent en retour sa propre position au sein du champ artistique ${ }^{39}$. La diffusion de ce programme aura pour conséquence notable l'élargissement considérable de l'audience de Ziyad Rahbani, cette dernière s'affranchissant peu à peu de toute appartenance communautaire et obédience politique.

36. «Zawāj al-mawqif al-siyāsì min al-mawqif al-fannī wa al-thaqāfì ». BASHA 2005, p. 22.

37. BARDAWIL 2002, p. 69.

38. Pour une analyse détaillée du contenu de l'émission, voir BARDAWIL 2002, p. 70-86.

39. Il critique simultanément des postures artistiques aussi opposées que celle de Mounir Abou Debs et celle du théâtre des Chansonniers. cf. BARDAWIL 2002, p. 78. Le premier, comédien et metteur en scène, est généralement considéré comme le "père du théâtre libanais ». Son travail expérimental prend dans les années 1970 une tournure mystique rapprochant ses travaux de ceux de Grotowski. À l'encontre de cette tendance qui néglige les attentes du public se trouve le théâtre de cabaret dont les saynètes politiques attirent un public de plus en plus nombreux depuis la création de la première troupe de chansonniers en 1963. 


\section{Le reflet d'une époque}

En adéquation avec les attentes des milieux intellectuels et artistiques engagés de l'époque, l'engagement politique et la conception de l'art de Ziyad Rahbani participent à une logique de renversement des valeurs dominantes dans les domaines du spectacle.

Son souci de représenter la réalité sociale procède clairement d'une réflexion sur ce qu'on appelle la « réalité vécue ${ }^{40}$ ». C'est alors une composante essentielle de la production artistique engagée qui est l'objet de recherches concernant la portée de son utilisation dans les différentes disciplines artistiques. La troupe du Hakawâtî, par exemple, insiste dans son manifeste sur le fait que le sujet des créations doit «s'appuyer avec précision sur une réalité vécue, connue soit par l'expérience, soit grâce à des entretiens, soit encore par la recherche de documents ${ }^{41}$ ». Pour Ziyad Rahbani, cette « réalité vécue » est une source d'inspiration primordiale : l'attaque de la Bank of America lui a inspiré la trame de Nazil al-surūr et le « snack-bar» de Bi-l-nisba la-bokrā shū ? (Et pour demain alors?, 1978, 1993) évoque étrangement son environnement professionnel lorsqu'il travaillait comme pianiste dans différents bars de la capitale. Elle transparaît également chez lui à travers l'emploi d'un dialecte stylisé dont il puise le lexique dans les conversations quotidiennes.

Par ailleurs, son refus de travailler avec des acteurs professionnels participe d'un rejet généralisé du régime de production commercial qui s'appuie sur le vedettariat. En outre, les membres de ses projets de création font avec lui de nombreux efforts pour parvenir à réduire le prix des places de manière à toucher un large public ${ }^{42}$. Ces préoccupations sont alors centrales pour les artistes engagés et la distance que ces derniers prennent avec les logiques économiques devient une preuve de leur engagement.

Enfin, l'accueil bienveillant de la critique spécialisée n'est certainement pas étranger à un certain nombre de valeurs que l'auteur partage avec celle-ci et parmi lesquelles se trouve la place centrale accordée au texte. Nazil alsurūr est ainsi l'objet de nombreux articles dans la presse libanaise. Si les critiques sont partagés sur son contenu politique, sa « qualité » est en revanche

40. En arabe, al-wāqi' al-mu' 'āsh.

41. Bayān masrah al-Hakawātī, p. 122. La troupe de théâtre du Hakawâtî (en français le Théâtre du Conteur) est fondée en 1977 par le metteur en scène et comédien Roger Assaf. Dans le manifeste que la troupe publie en 1979, on insiste essentiellement sur le refus du théâtre dominant et la recherche de nouvelles formes d'expression dramatique à travers la mise en cause du rapport à la production théâtrale et celle de la relation scène/salle.

42. HaWfar 1980, p. 91-92. 
largement saluée ${ }^{43}$. Issam Mahfouz, écrivain de théâtre et critique, voit en elle « la confirmation d'un nouveau talent ${ }^{44}$ ». Il est intéressant de relever à cet égard la position marginale qu'occupe l'écrivain de théâtre au sein du champ théâtral libanais.

Sans que soit remise en cause l'importance du texte, les membres du mouvement théâtral ne conçoivent que rarement sa production comme le fruit d'un travail littéraire. Pour le metteur en scène Roger Assaf par exemple, la composition du texte est « une part inséparable de la création théâtrale, il ne la précède donc pas ${ }^{45} »$. Dans cette perspective, l'écriture dramatique perd clairement son caractère littéraire. Par ailleurs, en arabe, l'écrit et l'oral sont clairement distingués. Si d'un côté l'arabe littéraire se prête mal à la spontanéité que requiert la scène, de l'autre, le dialecte est globalement déprécié d'un point de vue littéraire. Face à cette contradiction, les hommes de théâtre libanais adoptent différentes postures. La création collective, basée sur un travail d'improvisation et à laquelle Ziyad Rahbani s'oppose, est l'une des options retenues. Dans ce cas, le travail de l'écrivain de théâtre se réduit souvent à la fixation par écrit du travail collectif d'improvisation ${ }^{46} . A$ contrario et en marge de la création, le statut d'auteur de théâtre confère une légitimité accrue dans le cadre de la critique. Ainsi, au vu du rôle fondamental que Ziyad Rahbani accorde à l'auteur dans la composition de l'œuvre, on peut comprendre que celui-ci ait été l'objet d'un investissement particulier de la part de ceux qui voyaient dans l'écriture du texte le fondement de la création théâtrale.

De nombreuses caractéristiques démarquent pourtant cet auteur de l'« avant-garde » théâtrale.

\section{Une position ambiguë dans le champ théâtral}

\section{Origines sociales et formation théâtrale}

Contrairement à la plupart des hommes de théâtre libanais, Ziyad Rahbani n'a pas suivi d'études universitaires. Il a passé sa scolarité à Notre-Dame de Joumhour, dont les rangs sont essentiellement constitués d'élèves de la haute bourgeoisie chrétienne et francophone. La famille Rahbani, poussée par le

43. Sur les interprétations contrastées auxquelles a donné lieu Nazil al-surūr, voir BARDAwIL 2002, p. 64-68

44. Mahfouz 1974.

45. Assaf 1984, p. 11.

46. Issam Mahfouz et Henri Hamati, écrivains de théâtre publiés, ont tous deux expérimenté ces procédés d'écriture dans le cadre des activités de la troupe de l'Atelier dramatique de Beyrouth. 
succès phénoménal de Feyrouz et des Rahabneh, est en situation d'ascension sociale, mais reste de souche modeste ${ }^{47}$. Ziyad Rahbani est alors l'un des rares élèves dont la langue maternelle est l'arabe, ce qui le marginalise aux yeux des élèves et du corps enseignant, d'autant plus qu'il ne cache pas ses ambitions musicales. Son isolement à l'école ainsi que celui qu'il vit au sein du foyer familial le poussent très tôt à quitter la maison pour travailler en tant que pianiste dans différents bars de Beyrouth. Il n'obtient pas son baccalauréat. Cette situation de " dissonance ${ }^{48}$ » vécue dans le milieu scolaire n'est sans doute pas étrangère aux dispositions précoces qu'il développe à l'égard de l'engagement politique. Agnès Favier signale, à propos des «logiques de l'engagement », que la « dissonance » offre « des nouvelles représentations du monde qui, en tension avec les valeurs véhiculées dans le milieu familial, créent des situations de traumatisme ou d'injustice. Ces nouvelles situations nécessitent en retour d'apporter des réponses et de faire des choix, voire engendrent des accommodements ou des ruptures avec d'anciennes dispositions ${ }^{49}$. \ Les débuts de Ziyad Rahbani sur la scène libanaise sont, à partir de Nazil al-surūr, ponctués d'entretiens dans la presse qui révèlent ses tensions avec le milieu familial et le milieu scolaire ${ }^{50}$.

Ziyad Rahbani n'a pas non plus suivi de formation théâtrale. Or, dans le courant des années 1970, le mouvement théâtral est en pleine structuration. À l'initiative de la première génération d'hommes de théâtre, le mouvement se dote en effet d'institutions, publiques et privées, destinées à la formation d'une nouvelle génération de praticiens. De fait, les nouveaux entrants dans le monde du théâtre sont tous issus de centres de formation locaux ou étrangers. Ils ont ainsi acquis au cours de leur formation une culture particulière, liée aux conventions professionnelles du théâtre ${ }^{51}$, aisément identifiable à leurs références théoriques.

A contrario, Ziyad Rahbani dénie à son travail de création toute référence théorique. L'auteur réplique régulièrement que son seul bagage en la matière se résume à un ouvrage de Stanislavski et que sa connaissance de Brecht ne se fonde que sur la lecture d'articles et dossiers publiés dans la presse. Il dément ainsi toute tentative d'application des procédés de la distanciation brechtienne dans son œuvre ${ }^{52}$. En outre, sa conception de l'écriture dramatique est aussi

47. Avant de devenir l'illustre poète et compositeur que l'on sait, 'Assi Rahbani était gardien de la paix.

48. « Être en dissonance, c'est d'abord se sentir étranger à son univers scolaire, soit culturellement soit socialement. Cette marginalisation n'est bien souvent que le prolongement d'une situation atypique de la famille dans son environnement socio-communautaire. » FAVIER 2005, p. 357.

49. FAVIER 2005, p. 357.

50. Dakroub et SAad 1985, p.101. Bardawil 2002, p. 39-43.

51. BECKER 1988.

52. Dakroub et SAAD 1985, p.105. 


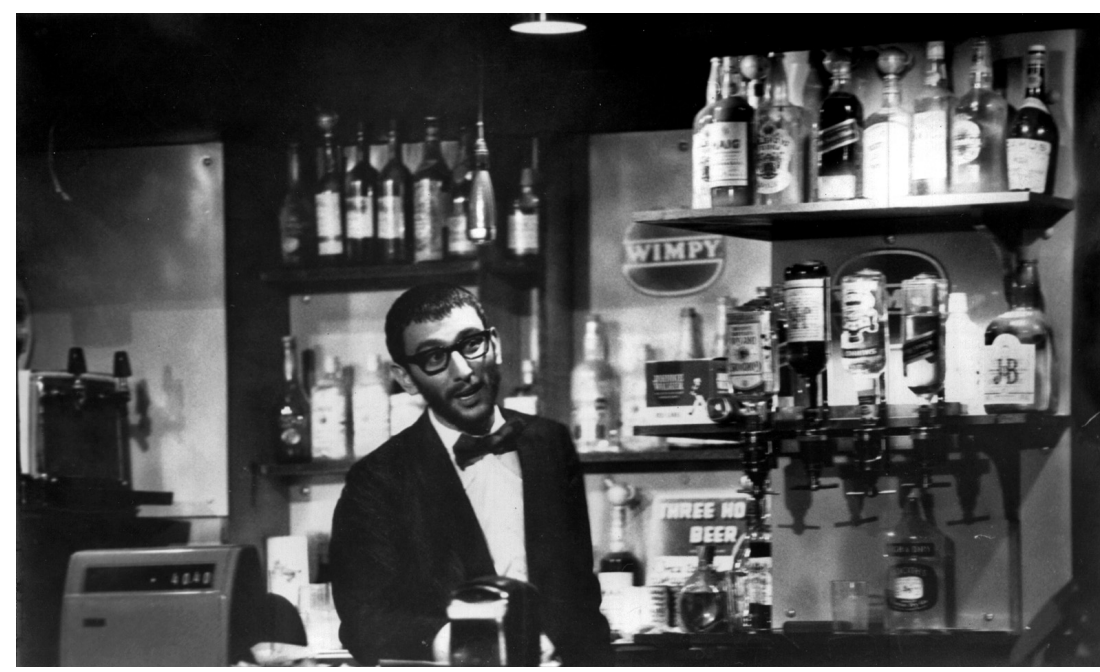

Ziyad Rahbani dans Bi-l-nisba la-bokrā shū? (1978). (C) Dār al-mukhtarāt.

problématique. Il déclare en effet que l'écriture du texte ne commence chez lui qu'après la mise en place du décor, étape qui lui permet d'évaluer avec précision la longueur des phrases qu'il compose ${ }^{53}$. Aux yeux des metteurs en scène libanais, les « ficelles » dont use Ziyad Rahbani pour monter ses pièces sont pour le moins « discutables tant elles paraissent rudimentaires au point de vue scénographique ${ }^{54}$. $\gg$ En ce sens, il fait figure d'anti-théoricien au sein du mouvement théâtral libanais, ce qui le place en nette opposition par rapport à l'《 avant-garde ».

\section{En opposition avec l'« avant-garde»}

La naissance puis l'évolution d'un «théâtre d'art» au Liban se sont essentiellement appuyées sur la figure du metteur en scène et, dès les débuts du mouvement, on constate la quasi-absence d'auteurs dramatiques dans la production scénique. Le rapport des hommes de théâtre aux formes d'arts consacrées - essentiellement la poésie et la littérature - est alors ambivalent. Bien que le texte maintienne le lien entre l'art dramatique et la création littéraire, ce sont les procédés scéniques qui sont retenus comme critères spécifiques d'appréciation de la création théâtrale. Même si les enjeux de l'autonomisation de cet art nouveau sont forts, face à la littérature notamment, ce phénomène ne saurait être entendu uniquement comme le fruit d'une stratégie de distinction

53. Dakroub et SAAD 1985, p. 105-106.

54. Abou Mourad 2002, p. 679. 
des nouveaux entrants qu'incarnent les hommes de théâtre dans le champ culturel ; le primat de la scène résulte aussi de la convergence de différents facteurs liés à la fois à une évolution du champ littéraire national et à celle des conceptions de la dramaturgie en Europe.

Sur le plan local et en prolongement de la "révolution structurelle » du champ littéraire ${ }^{55}$, on observe l'intérêt croissant des hommes de lettres libanais pour le théâtre. Celui-ci se cristallise notamment dans un effort de traduction du répertoire dramatique occidental. Cet effort se double de la volonté d'adapter l'arabe littéraire aux exigences de la scène et, à travers elle, au public ${ }^{56}$. À cette fin, on œuvre à une forme d' « oralisation » de la langue littéraire, soit par allègements syntaxiques, soit par rapprochement avec le dialecte, soit encore par la « libanisation » des œuvres adaptées ${ }^{57}$. La mise en œuvre de ces divers procédés illustre clairement le nouvel intérêt des hommes de lettres libanais pour la pratique de la scène.

La primauté accordée à la scène doit également être appréhendée dans une évolution globale du répertoire contemporain. Dès ses débuts, le théâtre libanais côtoie un ensemble de textes et de théories liés aux transformations du théâtre européen ${ }^{58}$. Ce dernier est confronté, depuis le début des années 1950, à «une crise de la littérature théâtrale ${ }^{59}$ ». Depuis l'apparition du théâtre de l'absurde en tant que genre (contenu et forme), l'intervention massive du discours didascalique signale la conscience chez les auteurs de théâtre de l'importance de ce qui échappe à la parole. En ce sens, ces textes contemporains au contenu visuel explicite éloignent le texte théâtral de sa définition classique de "poème dramatique » et le posent d'emblée comme partition pour la représentation ${ }^{60}$. La découverte des écrits d'Artaud et de Brecht concourt également à relativiser l'importance du texte.

La convergence de ces facteurs contribue donc à assurer la prédominance du metteur en scène au sein du mouvement théâtral libanais et, lorsque Ziyad Rahbani affirme la prééminence du texte, déniant tout rôle à la mise en scène,

55. La révolution littéraire des années 1960 est symboliquement marquée par la fondation quasi simultanée de la revue Shi $r$ (Poésie) et de la première Association des artistes (Jam 'iyyat al-fannāninn) en 1957.

56. Ce souci partagé des avant-gardes littéraires et théâtrales est incarné par la participation au mouvement de traduction de personnalités telles qu'Adonis, Ounsi al-Hajj ou Émile al-Boustani.

57. La « libanisation » d'une œuvre procède à la fois de sa traduction vers le dialecte libanais et de son adaptation à un contexte local.

58. La plupart des hommes de théâtre libanais montrent, en revendiquant des postures dramaturgiques claires, leur connaissance des diverses conceptions dramaturgiques en vogue dans le théâtre occidental. Il existe en outre une littérature théâtrale francophone qui favorise, à travers sa production, la connaissance de ces nouveaux courants. Mentionnons les noms de Georges Shéhadé et de Robert Abirached.

59. Proust 2001, p. 474.

60. ZARAGOZA 2005. 
qu'il décrit dans son théâtre comme une « exécution littérale du texte ${ }^{61}$ », il se place bien à contre-courant de ce mouvement. Sa marginalité est encore renforcée par son respect des principes de la dramaturgie classique, liés à la scène à l'italienne, comme la règle des trois unités. Toutes les recherches menées dans le cadre du mouvement théâtral visent justement à mettre en cause ces règles considérées comme des principes réactionnaires et bourgeois suscitant la passivité du public.

\section{Personnage public et notoriété}

Ziyad Rahbani se distingue encore des pratiques en vogue dans le champ théâtral libanais par une visibilité inhabituelle dans l'espace des productions culturelles, et ce d'autant que sa notoriété s'appuie sur des principes de reconnaissance et de diffusion étrangers au monde du théâtre.

Ainsi, la diffusion de l'émission Ba 'dnā țayyibin ... qul Allah ! a un impact retentissant sur le public. Outre cette émission, ses interventions répétées sur les ondes ont perpétué et renforcé son rapport de proximité avec une frange importante de la population libanaise, notamment parmi la jeunesse ${ }^{62}$. Au cours de sa carrière, Ziyad n'a jamais cessé de composer ${ }^{63}$, ce qui a naturellement favorisé sa reconnaissance par un public large.

Dans le domaine du théâtre, la réduction de son langage dramatique à un travail sur le texte, parce qu'elle a rendu possible l'enregistrement de ses pièces puis leur diffusion sur support audio, a largement favorisé la connaissance de son œuvre auprès d'un public étranger à la fréquentation des salles de théâtre. Notons que ces enregistrements s'opposent aussi radicalement à la conception répandue de l'œuvre d'art rare, parce que non stockable et non reproductible ${ }^{64}$, conception implicitement accréditée au Liban par la primauté accordée à la recherche scénique, éphémère par essence.

La diversité de ses compétences et sa très large audience le poussent vers une forme de consécration proche de celle du vedettariat. Cela peut paraître paradoxal, car, comme on l'a vu, Ziyad Rahbani tente de s'opposer à ce système. D'une part, en tant qu'auteur, compositeur et comédien, il est

61. En arabe tanfidh harfi li-l-ma 'āni, DAKROUB et SAAD 1985, p. 111.

62. Toutes ses émissions de radio sont disponibles en cassettes et $\mathrm{CD}$. Il est en outre régulièrement invité sur les plateaux de télévision.

63. Outre les musiques et chansons composées et écrites pour Feyrouz et pour ses propres pièces, on lui doit notamment Bi-l-afrah (1977), Anā mush käfir (1985), Hudū'nisbï (1985), Bimā enno (1995), avec la chanteuse libanaise Salma Monodose (2001) et, plus récemment, Ma lāmūt akida (2007) pour la chanteuse tunisienne Latifa. Il est par ailleurs le compositeur de la bande originale des films Le cerf-volant (2003) et Civilisées (1998) de Randa Chahal-Sabbagh.

64. Moulin 1995. 
toujours au premier plan dans ses créations théâtrales et y incarne d'ailleurs systématiquement le premier rôle. Ziyad Rahbani a de fait créé son propre public et c'est généralement le talent de l'auteur-comédien que l'on vient applaudir au théâtre ${ }^{65}$. D'autre part, à l'instar des formes dramatiques les moins consacrées dans le champ, le succès du théâtre de Ziyad Rahbani doit beaucoup à la popularité des personnages simples et sans instruction qu'il incarne. Les hiatus qu'engendre leur incapacité à saisir pleinement les enjeux des situations dans lesquelles ils sont pris ont un potentiel comique aisément exploitable. Certains ont ainsi pu le comparer à Buster Keaton ${ }^{66}$. Ce type de procédés rapproche immanquablement ses œuvres du théâtre commercial des Chansonniers ou encore de celui du Théâtre national et de sa vedette Chouchou ${ }^{67}$.

Enfin, l'opposition récurrente dans ses pièces entre le bon sens populaire et la finesse de l'instruction relève finalement du poncif. Sous l'emphase, c'est toujours la vacuité du discours qui ressort avec éclat. La figure de l'intellectuel - soit un politicien (Nazil al-surūr, Fìlm amirkì tawìl ${ }^{68}$ ), soit un poète (Bi-l-nisba la-bokrā shū ?), soit encore un journaliste (Shi fāshil) - est ainsi systématiquement opposée aux personnages naïfs et ingénus qu'incarne l'auteur. Sans vouloir dénier à cette constante sa dimension critique et politique, l'opposition permanente de ces deux types de personnage reflète de manière troublante la trajectoire professionnelle de l'auteur et peut enfin traduire « les tensions intériorisées de sa position sociale ${ }^{69} »$. Il ne s'agit pas pour autant de dénier à ses pièces leur portée critique, ni de porter atteinte à l'acuité des analyses de l'auteur, en ne voyant dans ces dernières qu'un reflet de sa trajectoire sociale. Mais le déplacement du point de vue critique permet de situer la spécificité de l'œuvre ailleurs, au carrefour entre l'état transitoire du champ théâtral libanais et les dispositions sociales de l'auteur, et ainsi de mieux comprendre la place exceptionnelle et paradoxale que son théâtre occupe dans l'histoire du mouvement théâtral libanais.

Ainsi, malgré l'ascension sociale de la famille Rahbani, l'échec scolaire de Ziyad interrompt le processus par lequel il serait passé du statut d'artiste à celui de lettré ou d'intellectuel, rang dont la majorité des hommes de théâtre

65. Les comédiens qui ont travaillé avec Ziyad Rahbani précisent que son public large leur permet de vivre correctement de chaque création. HAWFAR 1980, p. 91-92.

66. Traboulsi 1993.

67. Hassan 'Ala al-Din, dit « Chouchou », est une autre personnalité atypique du théâtre libanais. En 1965, il fonde, en collaboration avec Nizar Miqati, le Théâtre national (al-Masrah al-wațani), qui devient le premier théâtre permanent du Liban. Ce théâtre, dit aussi théâtre de Chouchou (Masraḥ Shūshū), repose essentiellement sur ses qualités d'acteur et on le considère généralement comme le père du Boulevard libanais.

68. Long métrage américain $(1980,1993)$.

69. MARY 2006, p. 59. 
sont gratifiés, si l'on juge de leur niveau d'instruction (diplômes universitaires élevés) et de leur production écrite (publiée sous forme d'ouvrages ou dans des revues littéraires ou scientifiques de prestige). Son public ne s'y trompe pas lorsqu'il voit dans les personnages qu'il incarne le reflet de sa propre personnalité ${ }^{70}$. Il n'est par ailleurs pas un entretien où l'ambiguïté de son statut ne transparaisse : reconnu pour l'importance de son œuvre théâtrale au cours de la guerre, il dénie systématiquement toute compétence théorique dans le domaine du théâtre. Jouissant d'une notoriété aussi exceptionnelle que relative, son statut d'artiste l'attire parfois, mais toujours à son corps défendant, vers les sphères culturelles les plus élevées.

\section{Conclusion}

Observer la carrière artistique de Ziyad Rahbani présente certains avantages. Celle-ci nous éclaire tout d'abord sur les liens spécifiques qui unissent le théâtre et la chanson au cours de cette période. On observe une communauté de pratique qui unit les deux arts, renforcée par le partage de valeurs éthiques et idéologiques qui se cristallisent dans une aspiration à renverser le mode de production artistique de masse.

La position ambiguë de cet artiste au sein du champ théâtral reflète, en outre, un état bien précis du mouvement théâtral. D'une part, la quête identitaire à laquelle semblait voué le théâtre libanais atteint son apogée lorsque Ziyad Rahbani apparaît sur la scène. L'interrogation de la réalité sociale emprunte des voies novatrices et les réflexions que suscite l'adaptation des concepts de la dramaturgie occidentale moderne à la réalité libanaise ou arabe aboutissent à des expérimentations fructueuses qui donnent ses lettres de noblesses au théâtre libanais. D'autre part, au cours de cette phase de structuration, le mouvement voit aussi la création d'institutions propres au théâtre. Le travail de délimitation de l'activité théâtrale est cependant interrompu par la crise sociale et politique qui agite le Liban et les institutions se voient parfois concurrencées par des organisations politiques. Cette période de transition rend ainsi possible l'entrée et la consécration d'artistes non professionnels, tels que Ziyad Rahbani, dans le champ théâtral.

Par ailleurs, l'étude de la trajectoire professionnelle des artistes libanais montre qu'au cours de cette période, l'engagement politique est un facteur d'intégration au champ artistique. Pour Ziyad Rahbani, celui-ci marque aussi une rupture avec le langage dramatique familial et favorise son apparition en tant qu'acteur indépendant sur la scène libanaise. Son engagement politique

70. Kamal Abou Ghaida 2002. 
a plusieurs dimensions. Dans le domaine de l'art, il transparaît dans ce que Fadi Abdo Bardawil appelle « les manifestations du politique dans la vie de tous les jours ${ }^{71}$ » et participe d'une réflexion globale des milieux artistiques et intellectuels sur la «réalité vécue ». Dans la vie publique, ses prises de position en faveur du parti communiste et son installation à l'ouest de la capitale ont un impact certain sur la perception que le public a de l'artiste. Cependant, si l'engagement politique facilite l'intégration au monde de l'art, le champ artistique n'en reste pas moins traversé par des clivages esthétiques. Ces derniers, pour leur part, se révéleront un facteur de la marginalisation de Ziyad Rahbani dans le domaine du théâtre. Sa situation paradoxale et son discrédit sur le plan théâtral sont exemplaires de la contradiction inhérente entre avant-gardisme esthète et avant-gardisme politique décrite par Nathalie Heinich ${ }^{72}$.

Ce positionnement artistique ambivalent engendre des effets paradoxaux. Malgré son rejet de l'héritage théâtral de ses parents, il est parfois considéré comme leur plus digne héritier ${ }^{73}$. Par ailleurs, si son dédain affiché pour l'esthétique de la scène le marginalise dans le milieu du théâtre, la place centrale qu'il accorde à ses textes et leur enregistrement ont permis une diffusion de son œuvre tout à fait inhabituelle dans la production dramatique libanaise. Ainsi, non seulement le public a découvert et continue de découvrir Ziyad à travers son théâtre, mais ce dernier est en passe d'entrer dans le « répertoire national ». En effet, la quête d'identité et d'authenticité du mouvement théâtral libanais ayant rapidement détourné ce dernier du texte de théâtre pour le pousser à se consacrer à l'innovation scénique, le théâtre libanais souffre aujourd'hui d'une carence évidente. S'il existe bien un fonds commun d'œuvres dramatiques, il est largement méconnu du public et délaissé par les troupes et les metteurs en scène contemporains. En revanche, l'œuvre de Ziyad Rahbani se transmet d'une génération à l'autre et une référence, même furtive, à l'une de ses pièces souligne toujours la complicité des connaisseurs. De jeunes metteurs en scène revendiquent aujourd'hui son héritage et ses pièces sont régulièrement remises en scène à l'initiative de troupes d'amateurs ou dans le cadre d'activités scolaires. La transmission de l'œuvre sur support audio est cependant problématique. L'enregistrement fixe en effet une part non négligeable d'éléments scéniques (diction, rythme des pièces, musique

71. «The manifestations of politics in everyday life », BARDAWIL 2002, p. 88.

72. La sociologue voit une contradiction objective entre ces deux définitions de l'artiste idéal. « L'avant-gardisme esthète va de pair avec l'autonomisation des enjeux artistiques, tendant inévitablement à l'élitisme, tandis que l'avant-gardisme politique implique l'hétéronomie des enjeux, tendant au populisme voire à l'ouvriérisme. » HeINICH 2005, p. 302.

73. KASSIR 1997. 
et passages chantés interprétés sur scène) et pose notamment le problème du manque d'autonomie des textes par rapport à leur représentation d'origine et à la personnalité de l'auteur-comédien.

\section{Bibliographie}

Abou Mourad N., 2003 : al-Masrah al-lubnānì fi al-qarn al-'ashrīn (Le théâtre libanais au $X X^{e}$ siècle), Beyrouth.

Assaf R., 1984 : al-Masraha, aqni 'at al-madina (La théâtralité, les masques de la villes), Beyrouth, Dār al-muthallath li-l-tașmīm wa al-țibā'a wa al-nashr.

Assaf R., 2000 : Les multiples naissances du Théâtre libanais (http://www.alba.edu/ AR/fr/theatrelibanais.html).

BARDAwIL F.-A., 2002 : Art, War \& Inheritance: The aesthetics \& politics of Ziyad Rahbani, American University of Beirut.

Basha A., 2005 : Mawt mudìr masrah, Dhākira fi "al-ughniya al-siyāsiyya" (Mort d'un directeur de théâtre, souvenir de " la chanson politique »), Dār al-ādāb.

Bayān masrạ al-Hakawātì (Manifeste de la troupe du Hakawâtî), dans al-Hayāt al-masrahiyya, no 9, été 1979.

BeCKer H. S., 1988 : Les mondes de l'art, Paris, Flammarion.

Boltanski L., 1982 : Les cadres, la formation d'un groupe social, Paris, Éditions de Minuit.

Bourdieu P., 1992 : Les règles de l'art, genèse et structure du champ littéraire, Paris, Seuil.

DAKRoub M. et SAAD S., 1986 : « Ḥiwār ma'a Ziyād al-Raḥbānī ḥawla masīratihi al-masrahiyya : fĩ al-'alaqa bayn al-fann wa al-mawqif al-siyāsī » (Entretien avec Ziyad Rahbani autour de son langage dramatique : de la relation entre l'art et le positionnement politique), al-Taríq, Beyrouth, $\mathrm{n}^{\circ} 1$, février.

FAVIER A., 2004 : Logiques de l'engagement et modes de contestation au Liban. Genèse et éclatement d'une génération de militants intellectuels (1958-1975), Thèse de science politique, sous la direction de Yves Schemeil, Université Paul Cézanne - Aix-Marseille III.

Hawfar (AL-) N., 1980 : « Nadwa ma'a firqat Ziyād al-Raḥbānī » (Rencontre avec la troupe de Ziyad Rahbani), al-Hayāt al-masrahiyya, no 14, automne, p. 86-99.

Kamal Abou Ghaida S., 2002 : Analyzing the Ziyad Rahbani Phenomenon Through Fan Discourse, American University of Beirut.

Kassir S., 1994 : La guerre du Liban. De la dissension nationale au conflit régional, Beyrouth, Karthala-Cermoc.

Kassir S., 1997 : « La république familiale », L’Orient-Express, nº 24, novembre. 
KHoury J., 1999 : « Le théâtre arabe entre le țarab oriental et le drame conflictuel », Travaux et jours, 64, automne, p. 91-95.

MAhfouz I., 1974 : « Ziyād al-Raḥbānī fĩ Nazil al-surūr, ta'akkadat mawhiba jadīda » (Ziyad Rahbani dans Nazil al-surūr, un nouveau talent confirmé), al-Nahār, 20 novembre.

Mary P., 2006 : «Le cinéma de Jacques Tati et la politique des auteurs », ARSS, 161-162, p. 42-65.

Mermier F., 2005 : Le livre et la ville. Beyrouth et l'édition arabe, Arles, Actes Sud/ Sindbad, coll. Hommes et sociétés.

Moulin R., 1995 : «La genèse de la rareté artistique », dans R. Moulin (éd.), De la valeur de l'art, Paris, Flammarion, p. 161-191.

PiCARD É., 1988 : Liban, État de discorde. Des fondations aux guerres fratricides, Paris, Flammarion.

Picaudou N., La déchirure libanaise, Bruxelles, Éditions Complexes, coll. Questions au $\mathrm{XX}^{\mathrm{e}}$ siècle, 1989.

Proust S., 2001 : «Une nouvelle figure de l'artiste, le metteur en scène de théâtre ", Sociologie du travail, vol. 43, no 4, p. 471-489.

Salamé Gh., 1974: Le théâtre politique au Liban (1968-1974), Beyrouth, Dar el-Mashrek éditeurs, Publications du centre culturel universitaire, coll. Hommes et sociétés du Proche-Orient, 7.

SAPIRO G., 1999 : La guerre des écrivains, 1940-1953, Paris, Fayard.

Traboulsi F., 1993 : «Le phénomène Ziyad Rahbani », Méditerranées, 5, p. 115-118.

Zaragoza G., 2005 : «Le statut du texte théâtral », dans G. Zaragoza et al. (éd.), Études théâtrales, Paris, PUF, coll. Quadrige.

\section{Pièces et œuvres citées}

\section{Atelier dramatique de Beyrouth}

Majdalūn, 1969, publiée sans date et sans maison d'édition.

\section{Jalal Khoury}

Wayzmānū, Bin Ghüryūn wa Shurakāhu, 1968, non publiée.

\section{Issam Mahfouz}

al-Qatl, Beyrouth, Dār al-fikr al-jadīd, 1968.

\section{Les frères Rahbani}

al-Maḥațta, 1973, in 'Āsì wa Manșūr al-Raḩbānì, al-a 'māl al-masrahiyya al-kāmila, Jounieh, Dynamic graphic li-l-țibā'a wa al-nashr, 2003 (disponible en CD et DVD). 


\section{Ziyad Rahbani}

Théâtre

Sahriyya, 1973, non publiée (disponible en CD et DVD).

Nazil al-surūr, 1974, Beyrouth, Dār al-mukhtarāt, 1993 (disponible en CD).

Bi-l-nisba la-bokrā shū ?, 1978, Beyrouth, Dār al-mukhtarāt, 1993 (disponible en CD).

Fìlm amirki țawìl, 1980, Beyrouth, Dār al-mukhtarāt, 1993 (disponible en CD).

Shì fāshil, 1982, Beyrouth, Dār al-mukhtarāt, 1993 (disponible en CD).

Bi-khusūs al-karāma wa al-sha'b al-'anìd, 1993, non publiée (disponible en CD et DVD).

Law la fushat al-amal, 1994, non publiée, (disponible en CD et DVD).

Radio (disponibles en CD)

Ba'dnā țayyibin... qul Allah!, 1976. L'intégralité de l'émission est accessible sur le site «alMashriq" (http://almashriq.hiof.no/lebanon/700/780/ziad-rahbani/ baadna-taybeen/index.html)

al-'aql zina (La raison est un luxe), 1986.

Shì tābi 'li-shì tābi 'shì (La suite de la suite), 1987.

Musique

Bi-l-afrah (album, 1977).

Anā mush kāfir (album, 1985).

Hudū' nisbi (album, 1985).

Bimā enno (album,1995).

Monodose (album avec Salma, 2001).

Ma 'lūmāt akida (album avec Latifa, 2006)

Avec Feyrouz

Waḥdun (album, 1979)

Ma 'riftī fik (album, 1985)

Kïfak enta (album, 1991)

Ilā 'Āsì (album, 1995)

Mish Kāyen heik tkūn (album, 1999)

Walā kif (album, 2002) 
